\title{
Efecto citotóxico de Deoxinivalenol sobre la proliferación de la línea celular HepG2
}

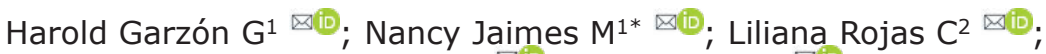 \\ Siham Salmen $\mathrm{H}^{3} \bowtie\left(\mathbb{D} ;\right.$; Manuel Gil $\mathrm{D}^{4} \otimes(\mathbb{D})$
}

\begin{abstract}
${ }^{1}$ Universidad de Pamplona, Facultad de Ciencias Básicas, Departamento de Biología, Grupo de Investigación en Biología Molecular y Genética (BIOMOGEN), Km 1 Vía Bucaramanga, Ciudad Universitaria, Pamplona, Colombia.

2Universidad de Pamplona, Facultad de Ciencias Básicas, Departamento de Microbiología, Grupo de Investigación en Microbiología y Biotecnología (GIMBIO), Km 1 Vía Bucaramanga Ciudad Universitaria, Pamplona, Colombia.

${ }^{3}$ Universidad de Los Andes, Facultad de Medicina, Instituto de Inmunología Clínica (IDIC), Edificio Louis Pasteur, Mérida, Venezuela. 4Universidad de Pamplona, Facultad de Ciencias Básicas, Departamento de Matemáticas, Grupo de Investigación en Biología Molecular y Genética (BIOMOGEN), Km 1 Vía Bucaramanga, Ciudad Universitaria, Pamplona, Colombia.

*Correspondencia: najame6@gmail.com
\end{abstract}

Recibido: Julio 2020; Aceptado: Marzo 2021; Publicado: Agosto 2021.

\section{RESUMEN}

Objetivo. Determinar el efecto citotóxico e inducción de la apoptosis de Deoxinivalenol (DON) sobre la línea celular de hepatocarcinoma humano (HepG2). Materiales y métodos. La línea celular HepG2 se expuso a concentraciones de 10, 25, 50 y $75 \mu \mathrm{M}$ de DON liofilizado durante 48 y 72 horas. Posteriormente, la actividad citotóxica de DON se evaluó empleando el ensayo MTT (bromuro de 3-(4,5-dimetil-2-tiazolil) -2, 5-difeniltetrazolio). Finalmente, se analizaron los cambios morfológicos propios de la apoptosis en las células HepG2 por microscopía electrónica de transmisión, después del tratamiento con $50 \mu \mathrm{M}$ de DON durante 48 horas. Resultados. DON, afecta la actividad metabólica y proliferación de las células HepG2 por encima de los $10 \mu \mathrm{M}$, en comparación con el control. La concentración inhibitoria media $\left(\mathrm{CI}_{50}\right)$ de DON sobre las células HepG2, fue de 42.8 $\mu \mathrm{M} D E \pm 1.2$ y de $29.6 \mu \mathrm{M} \mathrm{DE} \pm 3.1$ a las 48 horas y 72 horas de tratamiento, respectivamente. Se observaron características morfológicas de la apoptosis en las células HepG2, como la fragmentación nuclear y celular, invaginación de la membrana plasmática y formación de los cuerpos apoptóticos. Conclusiones. DON, es un agente citotóxico sobre las células HepG2 que altera la actividad metabólica celular, con un efecto antiproliferativo significativo de manera dependiente a la concentración y al tiempo de exposición, e induce la muerte celular apoptótica.

Palabras clave: Fusarium spp.; proliferación; toxicidad (Source: DeCS).

\section{ABSTRACT}

Objetive. To determine the cytotoxic effect and induction of apoptosis of Deoxynivalenol (DON) on the human hepatocarcinoma cell line (HepG2). Materials and methods. The HepG2 cell line was exposed to concentrations of 10, 25, 50 and $75 \mu \mathrm{M}$ of lyophilized DON for 48 and 72 hours. Subsequently, the cytotoxic activity of DON was evaluated using the MTT assay. Finally, it analyzed the morphological changes of apoptosis in HepG2 cells by transmission electron microscopy, after treatment with $50 \mu \mathrm{M}$ of DON for 48 hours. Results. DON, affects the metabolic activity and proliferation of HepG2 cells 
above $10 \mu \mathrm{M}$, compared to the control. The $\mathrm{IC}_{50}$ of DON on HepG2 cells, $42.8 \mu \mathrm{M} \mathrm{SD} \pm 1.12$ and 29.6 $\mu \mathrm{M}$ SD \pm 3.1 at 48 hours and 72 hours of treatment, respectively. The morphological characteristics of apoptosis in HepG2 cells, such as nuclear and cellular fragmentation, invagination of the plasma membrane, and the formation of apoptotic bodies. Conclusions. DON is a cytotoxic agent in HepG2 cells that alters cellular metabolic activity, with a significant antiproliferative effect dependent on concentration and exposure time, and induces apoptotic cell death.

Keywords: Fusarium spp.; proliferation; toxicity (Source: CAB).

\section{INTRODUCCIÓN}

Deoxinivalelol (DON), es un tricoteceno tipo B producido por Fusarium spp. (1), sintetizado durante el crecimiento de Fusarium graminearum y Fusarium culmorum sobre granos de cereales, tales como trigo, cebada y maíz. A nivel mundial, es común la contaminación por tricotecenos en granos de cereales, subproductos de cereales, comida humana y alimentación animal; problema que cada vez es más frecuente, debido a que se da la colonización por Fusarium spp., por la práctica de siembra sin arado y por una inadecuada rotación de cultivos, además, de los cambios en los patrones climáticos en regiones templadas $(2,3,4)$.

Los tricotecenos, de bajo peso molecular ( $200-$ $500 \mathrm{Da}$ ), pueden difundirse rápidamente en las células (5). La naturaleza anfipática de DON le permite atravesar fácilmente las membranas celulares e interactuar con ribosomas, mitocondrias y retículo endoplasmático (6). Los efectos tóxicos de DON son múltiples a nivel de células eucarióticas, por ejemplo, DON interactúa con la enzima peptidil transferasa en la subunidad ribosomal 605 y desencadena estrés ribotóxico, además, varios estudios sugieren que induce la producción de radicales libres, ocasionando estrés oxidativo; mecanismos que causan daño celular, como es la inhibición de la síntesis de proteínas, ARN, y ADN; también, se afecta la función mitocondrial, la viabilidad celular, la proliferación, diferenciación celular y la integridad de la membrana, que finalmente, inducen la apoptosis o necrosis $(3,6,7)$.

El proceso de apoptosis avanza a través de una cascada compleja que involucra características morfológicas, moleculares y bioquímicas. Las características morfológicas de la apoptosis incluyen eventos tempranos como la contracción citoplasmática celular, disminución del volumen celular, condensación cromática y nuclear. A continuación, se presenta la fragmentación nuclear y, en consecuencia, la ruptura del ADN, la formación de ampollas en la membrana plasmática y la formación de cuerpos apoptóticos que son digeridos por fagocitosis por células vecinas o macrófagos, estos considerados eventos tardíos en el proceso de apoptosis. Además, en las etapas posteriores de la apoptosis, algunas de las características morfológicas incluyen modificación ultraestructural de los orgánulos citoplasmáticos, formación de vesículas con contenidos intracelulares, reordenamiento del citoesqueleto y pérdida de contacto con la matriz extracelular.

Estos cambios morfológicos se deben a eventos moleculares y bioquímicos característicos que ocurren en las células apoptóticas. Por ejemplo, dentro de los eventos bioquímicos iniciales está la externalización de fosfatidilserina en la mayoría de las células; esto imparte señales en las células apoptóticas, haciendo que sean absorbidas por los macrófagos y las células cercanas, especialmente in vivo. Los eventos bioquímicos adicionales incluyen la activación particular de las enzimas proteolíticas, entre ellas las caspasas iniciadoras y efectoras, que hidrolizan proteínas estructurales, además de las nucleasas que causan la fragmentación del ADN en sitios internucleosómicos, por un lado, y la inactivación de los sistemas de enzimas de reparación, por otro lado $(8,9,10)$.

En estudios in vitro de la toxicidad de DON se han utilizado cultivos primarios de tejidos de médula ósea, epitelial, hepático, linfoide, de riñón y pulmón; células consideradas sensibles a DON (11) además, de las células que hacen parte de los órganos que participan en la vía de absorción como el intestino y el hígado. Así mismo, la línea celular HepG2, derivada de un carcinoma hepatocelular humano, ha sido ampliamente utilizada en estudios in vitro, ya que mantiene funciones específicas del hígado y refleja el metabolismo de toxinas mejor que otras líneas celulares. Estudios del efecto de DON sobre HepG2, reportan que afectó la viabilidad 
celular al ser tratadas con una concentración de $0.9 \mu \mathrm{M}$, igualmente en otras células del epitelio intestinal, se inhibió la proliferación celular después de 24 horas de exposición a DON en un rango de concentraciones de 10-30 $\mu \mathrm{M}$ (1, $12,13)$. Otro estudio, obtiene concentraciones inhibitorias de DON para las células HepG2 de 1.0-15 $\mu \mathrm{M},(14)$. Por lo tanto, el objetivo de este trabajo de investigación fue determinar el efecto citotóxico de DON en la proliferación de la línea celular HepG2 a diferentes concentraciones y tiempos de tratamiento.

\section{MATERIALES Y MÉTODOS}

Micotoxina. Se efectuó la liofilización del estándar Micotox deoxinivalenol-HPLC en metanol (HPLC-DON-4), Micotox Ltda, Colombia. En resumen, se inició con la diálisis de la micotoxina DON en tampón Tris a $25 \mathrm{mM}$ durante 24 horas. Posteriormente, el contenido se traspasó a un tubo Falcon, se congeló durante 4 horas y se realizó el proceso de liofilización por 24 horas.

Células HepG2. La línea celular HepG2 (carcinoma de hepatoblastoma humano), se cultivó en RPMI-1640 suplementado con suero fetal bovino al $10 \%$ y con una solución de antibiótico al 0.05\%, hasta obtener una confluencia del $80 \%$. Posteriormente, las células se disociaron con tripsina $0.25 \%$ y se sembraron en placas de cultivo de 96 pozos, a una densidad de 20.000 células/100 $\mu$ por pozo. Las células, se dejaron durante 24 horas para la adhesión celular y luego se adicionó la micotoxina DON diluida DMSO (dimetilsulfóxido) a concentraciones de 5, 10, 25, 50 y 75 uM. Los cambios en la morfología y densidad celular se observaron bajo el microscopio óptico invertido Nikon 37762.

Proliferación de las células HepG2. El efecto de la micotoxina DON sobre la viabilidad celular, se determinó usando el ensayo MTT (bromuro de 3-(4,5-dimetil-2-tiazolil) -2, 5-difeniltetrazolio). Después de 48 y 72 horas de tratamiento con DON, se evaluó la viabilidad celular adicionando $100 \mu \mathrm{l}$ de MTT $(0.5 \mathrm{mg} / \mathrm{ml})$ y se incubó por 4 horas a $37^{\circ} \mathrm{C}, 5 \% \mathrm{CO}_{2}$ y $95 \%$ de humedad. Posteriormente, las placas se lavaron con PBS (tampón fosfato salino), y luego, se adicionó $100 \mu \mathrm{l}$ de isopropanol ácido para solubilizar los cristales de Formazán durante 15 minutos en condiciones de cultivo y en agitación suave por
5 minutos. Finalmente, se examinó la densidad óptica a $570 \mathrm{~nm}$ (espectrofotómetro Tecan Spectra classic) de la solución solubilizada (15) y se calculó el porcentaje de viabilidad celular.

Seguidamente, el efecto de la micotoxina sobre las células HepG2, se expresó como valores del porcentaje de inhibición usando la siguiente fórmula:

$\%$ Inhibición $=\left[1-\left(\frac{\text { Absorbancia media de células tratadas }}{\text { Absorbancia media del control }}\right)\right] \times 100$

La concentración inhibitoria, es decir, la concentración de la micotoxina necesaria para inhibir el $50 \%$ del crecimiento en las células HepG2 $\left(\mathrm{CI}_{50}\right)$, se midió mediante el cálculo de las curvas de inhibición de porcentaje y concentración. Se seleccionó el modelo no lineal hiperbólico para el ajuste de los datos. El análisis de regresión no lineal empleado permitió la obtención de los estimadores del modelo mediante mínimos cuadrados, usando como variable de respuesta la dosis inhibitoria y como variable de regresión la concentración de DON evaluados. Se utilizó este modelo por su gran utilidad en este tipo de ensayos y por tener el más bajo criterio de información de Akaike (AIC) (16).

Microscopía electrónica de transmisión. Se sembraron $3 \times 10^{6}$ células HepG2 en placas de cultivo durante 24 horas para permitir la adhesión celular, posteriormente, se adicionó 50 $\mu \mathrm{M}$ de DON durante 48 horas; a continuación, la monocapa celular se disoció con tripsina al $0.25 \%$, luego se lavó la suspensión celular con PBS y se centrifugó a $2.000 \mathrm{rpm}$ durante 10 minutos. EI precipitado celular se fijó por un período de 1 a 2 horas en una mezcla 3:3 (glutaraldehídoformaldehido al 3\% en tampón cacodilato 0.1 $\mathrm{M}, \mathrm{pH}$ 6.3). Después, la suspensión celular se centrifugó a $2.000 \mathrm{rpm}$ durante 10 minutos y se lavó con tampón cacodilato y se postfijó por 3 a 12 horas en una solución de tetraóxido de osmio al $1 \%$ preparado en el mismo tampón cacodilato. Concluido este tiempo, el precipitado celular se lavó con solución tampón y se deshidrató en concentraciones ascendentes de alcohol etílico seguido de óxido de propileno, subsiguientemente, se infiltró e incluyó en resina epoxídica. Del precipitado de células incluidas se realizaron secciones ultrafinas de 90 nanómetros de espesor, que fueron contrastadas con citrato de plomo y acetato de uranilo para el análisis ultraestructural de la apoptosis en el microscopio electrónico de transmisión ( $\mathrm{H}-7000$, Hitachi). 
Análisis estadístico. El análisis estadístico se realizó usando el software Graph Pad Prism para comparar las diferencias entre las medias del control y los grupos tratados con la micotoxina a las 48 y 72 horas. Los datos se analizaron con ANOVA de una vía y el test comparaciones múltiples de Dunnett's.

\section{RESULTADOS}

\section{Efecto de DON en la actividad metabólica de} HepG2. La actividad metabólica se observó en la capacidad de las células HepG2 para formar los cristales de Formazán. Las células HepG2 sin tratar con DON, presentaron alta actividad metabólica por la reducción del Bromuro de 3-(4,5-dimetiltiazol-2-ilo)-2,5-difeniltetrazol en cristales de Formazán (Figura 1A); mientras que, a medida que se incrementó la concentración de DON, esta actividad metabólica disminuyó en las células HepG2 (Figura 1B y 1C).

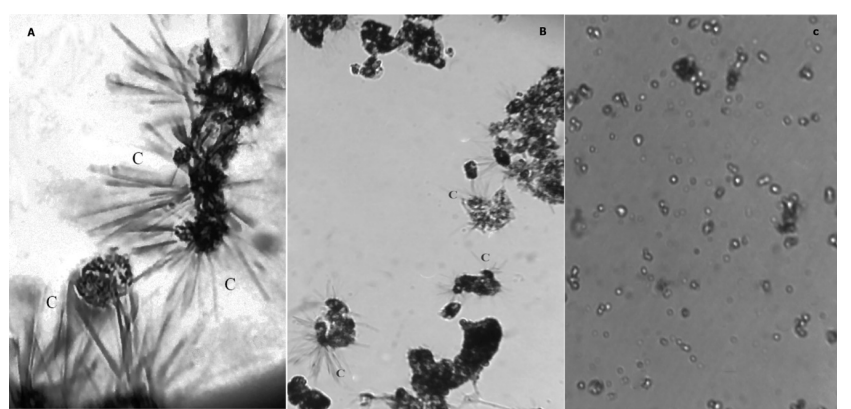

Figura 1. a. Células HepG2 sin tratar. b. Células HepG2 tratadas con una concentración media $(25 \mu \mathrm{M})$ de DON. c. Células HepG2 tratadas con una concentración alta $(75 \mu \mathrm{M})$ de DON. Aumento: A: 400X B y C: 200X. C= Cristales de Formazán. Microscopio óptico invertido Nikon 37762.

Efecto citotóxico de DON sobre las células HepG2. Los efectos citotóxicos de DON sobre la proliferación de células HepG2, se aprecian en la Figura 2. La proliferación celular, fue inhibida de forma dependiente de la concentración después de tratar las células HepG2 con DON en el rango de concentraciones de 0 a $75 \mu \mathrm{M}$ por 48 y 72 horas. Un efecto significativo de DON sobre la proliferación celular fue observado desde los $10 \mu \mathrm{M}(75.37 \% \mathrm{DE} \pm 4.0$ y $74.01 \%$
$D E \pm 0.66)$ en comparación con el control ( $98.2 \%$ $D E \pm 0.05)$ respectivamente $(p<0.0001)$. En consecuencia, a mayor concentración de DON (75 $\mu \mathrm{M}$ ) el porcentaje de proliferación fue reducido significativamente a $33.39 \%$ DE 0.0 y a $23.73 \%$ DE \pm 3.0 , observándose una disminución en la proliferación celular y en la actividad metabólica para la formación de los cristales de Formazán (Figura 1C). Por otro lado, los resultados, revelan un valor de la $\mathrm{CI}_{50}$ de $29.6 \mu \mathrm{M} D E \pm 3.1$ a las 72 horas de exposición a diferencia del valor de $\mathrm{CI}_{50}$ de $42.82 \mu \mathrm{M} \mathrm{DE} \pm 1.2$ a las 48 horas de exposición a DON.

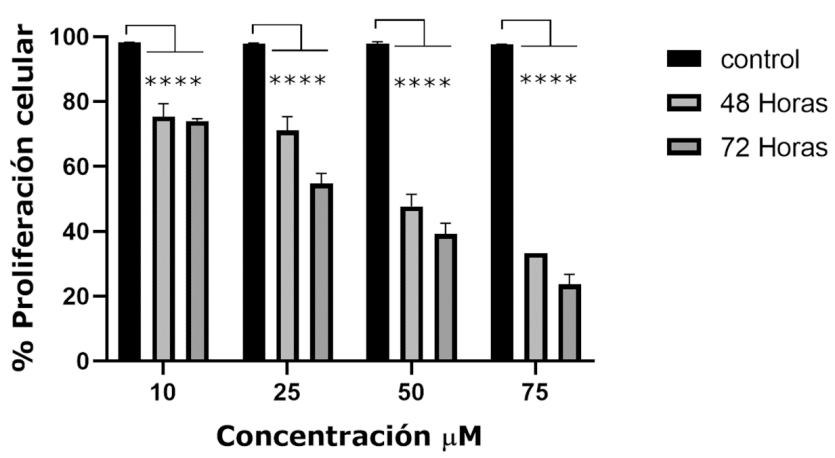

Figura 2. Proliferación de las células HepG2 tratadas con una concentración en un rango de 0 a $75 \mu \mathrm{M}$ de DON. Cada barra representa la media $\mathrm{DE} \pm(\mathrm{n}=3)$. **** $\mathrm{p}<0.0001$ versus el control usando ANOVA.

Apoptosis de las Células HepG2 por DON. Al analizar por microscopía electrónica de transmisión el proceso de apoptosis en las células HepG2 por la exposición a DON $(50 \mu \mathrm{M})$ por 48 horas, se observaron cambios en la morfología celular. En las células tratadas con DON se evidenció la fragmentación del contenido celular y nuclear, la formación de protuberancias en la membrana plasmática, cuerpos apoptóticos, alteraciones de la morfología celular y por tanto pérdida de los contactos célula a célula, características propias de células en apoptosis. Por otro lado, en el control, el núcleo mantuvo su forma redondeada con presencia de un nucléolo definido, la membrana nuclear y plasmática permanecieron intactas, y se observó un contenido celular y una morfología epitelial conservada en las células HepG2 (Figura $3 \mathrm{~A}$, B y C). 

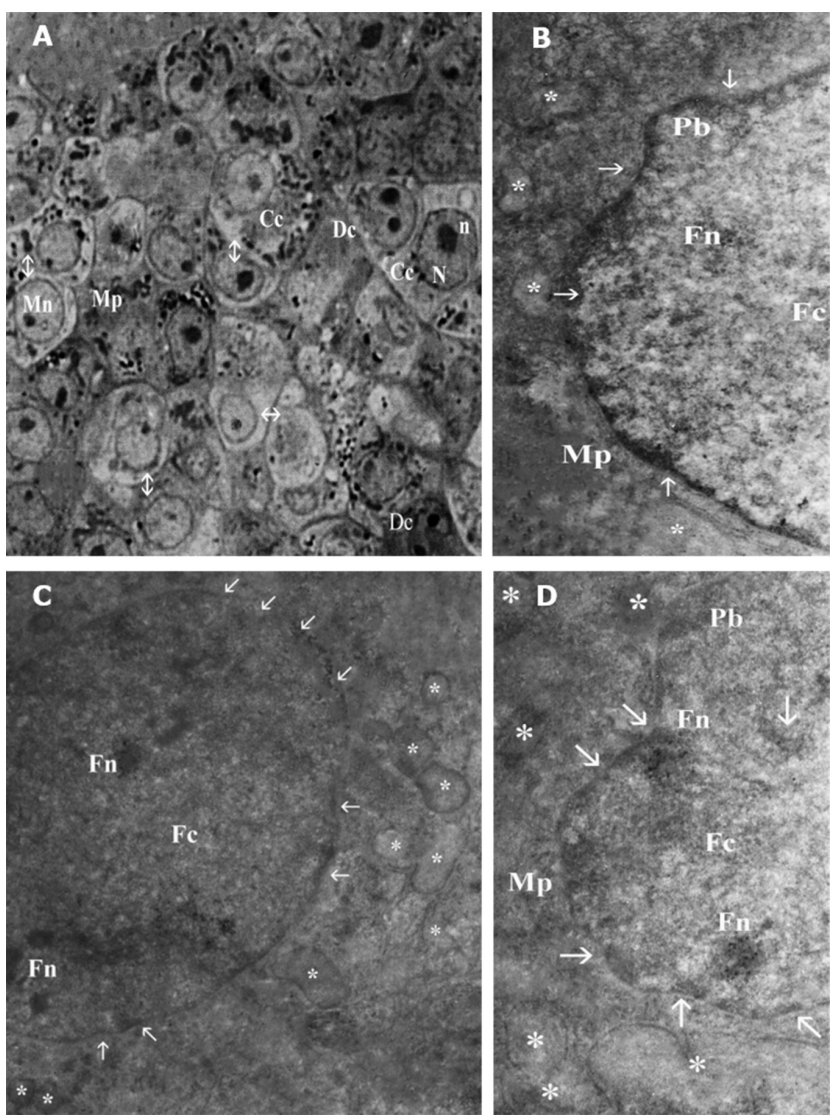

Figura 3. Microscopía electrónica de Transmisión de las células HepG2, expuestas a $50 \mu$ M DON. A. Control negativo, B - D. células expuestas a $50 \mu \mathrm{M}$ de DON. Aumento: A:4.000X B-D: 6.500X. $M n=$ Membrana nuclear, $M p=$ Membrana Plasmática, $\mathrm{Cc}=$ Contenido celular, $\mathrm{Dc}=$ División celular, $\mathrm{N}=$ Núcleo, $\mathrm{n}=$ Nucléolo, $\mathrm{Pb}=$ Protuberancia, $\mathrm{Fn}=$ Fragmentación nuclear, $\mathrm{Fc}=$ Fragmentación celular, $*=$ Cuerpos apoptóticos, $\rightarrow=$ Invaginaciones de la membrana plasmática, Flecha con doble punta $=$ Contacto célula a célula. Microscopio electrónico de transmisión (H-7000, Hitachi).

\section{DISCUSIÓN}

La citotoxicidad de DON ha sido estudiada tanto en modelos animales como en cultivos celulares debido a que las micotoxinas son contaminantes naturales de los alimentos y representan un factor de riesgo para la salud humana y animal. En el presente estudio se reporta por medio de ensayos in vitro el efecto citotóxico de diferentes concentraciones de DON sobre la línea celular HepG2.

El ensayo MTT, es una medida del metabolismo mitocondrial de las células, por lo que los resultados obtenidos, sugieren que la exposición a DON afecta este mecanismo celular en las células HepG2, dada la disminución en la formación de los cristales de Formazán a medida que aumentaban las concentraciones de DON. Así mismo, varios autores reportan que la exposición a DON provoca alteración metabólica en varias líneas celulares. Dentro de estos estudios, se encuentra que la exposición de las células de riñón embrionario humano (Hek-293) a concentraciones >7.5 $\mu$ M de DON, reduce significativamente la viabilidad celular, y disminuyen los niveles de NADH e incrementan los ROS, lo que puede estar relacionado con una disfunción mitocondrial (17).

Los resultados de citotoxicidad, revelaron una disminución de la proliferación de las células HepG2 en un 25.31\% DE \pm 0.96 , en la concentración más baja de $\operatorname{DON}(10 \mu \mathrm{M})$; no obstante, otros estudios reportan una disminución del $25 \%$ de las células HepG2 por la exposición a $1 \mu \mathrm{M}$ de DON (1), además, que la proliferación de células del epitelio intestinal es inhibida a concentraciones entre 10-30 $\mu \mathrm{M}$ de DON (3), lo que sugiere una menor sensibilidad de las células ensayadas a DON en este estudio, esta diferencia en los resultados puede ser debido a la pureza del patrón de DON probado en cada caso, en el presente estudio se utilizó un estándar de DON en etanol, que se liofilizó antes del tratamiento de las células HepG2.

En relación con el valor del $\mathrm{CI}_{50}$ de $42.82 \mu \mathrm{M}$ $\mathrm{DE} \pm 1.2$ obtenido luego de las 48 horas de exposición de las células HepG2 a DON, estos resultados difieren con los reportes actuales del valor de $\mathrm{CI}_{50}$ de 3.0 a $4.15 \mu \mathrm{M}$ a las 48 horas de exposición, utilizando las líneas celulares HepG2 y adenocarcinoma de colon humano ( $\mathrm{CaCo}-$ 2) y ensayos citotóxicos de rojo neutro y MTT $(14,18)$. En cuanto al tiempo de exposición de $72 \mathrm{~h}$, los resultados de $\mathrm{CI}_{50}$ obtenidos de 29.6 $\mu \mathrm{M} D E \pm 3.1$, en contraste a estos resultados, se encuentra que los valores de $\mathrm{CI}_{50}$ obtenidos en otras investigaciones a 72 horas de tratamiento de células HepG2 con DON fueron de $2.53 \mu \mathrm{M}$ $\mathrm{DE} \pm 0.21,4.30 \mu \mathrm{M} \mathrm{DE} \pm 0.36$ hasta $9.30 \mu \mathrm{M}$ por medio del ensayo MTT y Rojo Neutro $(14,19)$. Sin embargo, aunque las concentraciones del $\mathrm{CI}_{50}$ difieran entre autores, debido a las diversas condiciones experimentales evaluadas, así como la finalidad del estudio, tipo de células, tiempo de exposición, rango de concentración y ensayo citotóxico utilizado, pero se coincide en que el efecto de DON sobre la línea celular HepG2 es dependiente de la concentración y el tiempo.

Los cambios morfológicos en las células HepG2 expuestas a DON, evidenciados 
mediante microscopía electrónica, incluyeron la fragmentación del contenido celular y nuclear, invaginaciones de la membrana plasmática y formación de cuerpos apoptóticos, así como alteraciones de la morfología celular y por tanto pérdida de los contactos célula a célula (Figura 3: B-D), estos hallazgos indicaron que la citotoxicidad del DON en las células HepG2, se caracteriza por la muerte celular apoptótica. Dichos resultados coinciden con reportes, en donde observaron que las células epiteliales de tejido hepático humano (L-02) tratadas con DON, mostraron cambios morfológicos típicos de las células apoptóticas, con la aparición de núcleos de forma irregular y cromatina fragmentada, acompañado del aumento del estrés oxidativo con la generación de ROS y la disminución del potencial de membrana mitocondrial, además, con efectos tóxicos en la inhibición de síntesis de proteínas y ácidos nucleicos e inducción de la apoptosis (20). Otros estudios reportan lesiones apoptóticas en tejidos hepáticos de cerdos por la exposición a DON, revelando una condensación de la cromatina y cuerpos apoptóticos (21) y de igual manera la exposición a DON sobre las células de cáncer de colón humano HT-29 provoca la fragmentación nuclear y la presencia de cuerpos apoptóticos (22).

En conclusión, DON, ejerció un efecto citotóxico sobre las células HepG2 que alteró la actividad metabólica celular, con un marcado efecto antiproliferativo significativo de manera dependiente a la concentración y al tiempo de exposición; en consecuencia, induce una muerte celular apoptótica en las células HepG2, caracterizada por cambios morfológicos como la fragmentación del contenido celular y nuclear, invaginaciones de la membrana plasmática y la formación de cuerpos apoptóticos, así como alteraciones de la morfología celular, generando la pérdida del contacto célula a célula. Lo anterior, puede suponer que la alteración metabólica generada por DON, activa mecanismos celulares, posiblemente, mediante la vía mitocondrial, que desencadenan la apoptosis.

\section{Conflicto de intereses}

Todos los autores declaran que no existe ningún conflicto de intereses para la publicación del presente manuscrito.

\section{Agradecimientos}

A la Universidad de Pamplona por la financiación de esta investigación. Al Centro de Microscopía Electrónica "Dr. Ernesto Palacios Prü"; Instituto de Inmunología Clínica, Facultad de Medicina, Universidad de Los Andes, Mérida, Venezuela; Centro de Microscopía y Microanálisis, Bogotá, Colombia, por su apoyo y colaboración en el desarrollo de la investigación.

\section{REFERENCIAS}

1. Mayer E, Novak B, Springler A, SchwartzZimmermann $\mathrm{H}$, Nagl V, Reisinger $\mathrm{N}$, et al. Effects of deoxynivalenol (DON) and its microbial biotransformation product deepoxy-deoxynivalenol (DOM-1) on a trout, pig, mouse, and human cell line. Mycotoxin Res. 2017; 33(4):297-308. https://link.springer.com/article/10.1007/ s12550-017-0289-7

2. Pestka J. Toxicological mechanisms and potential health effects of deoxynivalenol and nivalenol. World Mycotoxin J. 2010; 3(4):323-347. https://doi.org/10.3920/ WMJ2010.1247
3. Pinton P, Tsybulskyy D, Lucioli J, Laffitte J, Callu P, Lyazhri F, et al. Toxicity of deoxynivalenol and its acetylated derivatives on the intestine: Differential effects on morphology, barrier function, tight junctions proteins and MAPKinases. Toxicol Sci. 2012; 130(1):180-190. https://www.ncbi.nlm. nih.gov/pubmed/22859312

4. Ren Z, Wang Y, Deng H, Deng Y, Deng J, Zuo $Z$, et al. Deoxynivalenol induces apoptosis in chicken splenic lymphocytes via the reactive oxygen species-mediated mitochondrial pathway. Environ Toxicol Pharmacol. 2015; 39(1):339-346. https://www.ncbi.nlm.nih. gov/pubmed/25553575 
5. Arunachalam C, Doohan F. Trichothecene toxicity in eukaryotes: Cellular and molecular mechanisms in plants and animals. Toxicol Lett. 2013; 217(2):149-158. https://www. ncbi.nlm.nih.gov/pubmed/23274714

6. Wu F, Groopman F, Pestka J. Public Health Impacts of Foodborne Mycotoxins. Annu Rev Food Sci Technol. 2014; 5:351-372. https:// www.ncbi.nlm.nih.gov/pubmed/24422587

7. Liao $Y$, Peng $Z$, Chen $L$, Nüssler A, Liu L, Yang W. Deoxynivalenol, gut microbiota and immunotoxicity: A potential approach? Food Chem Toxicol. 2018; 112:342-354. https:// www.ncbi.nlm.nih.gov/pubmed/29331731

8. Pistritto G, Trisciuoglio D, Ceci C, Garufi A, D'Orazi G. Apoptosis as anticancer mechanism: function and dysfunction of its modulators and targeted therapeutic strategies. Aging. 2016; 8(4):603-619. https://dx.doi. org/10.18632\%2Faging. 100934

9. Gordeziani M, Adamia G, Khatisashvili G, Gigolashvili G. Programmed cell selfliquidation (apoptosis). Annals of Agrarian Science. 2017;15(1):148-154. https:// www.sciencedirect.com/science/article/pii/ $\underline{\mathrm{S} 151218871630029 \mathrm{X}}$

10. Redza M, Averill D. Activation of apoptosis signalling pathways by reactive oxygen species. Biochimica et Biophysica Acta (BBA) - Molecular Cell Research. 2016; 1863(12):2977-2992. https://doi. org/10.1016/j.bbamcr.2016.09.012

11. Pestka J. Toxicological mechanisms and potential health effects of deoxynivalenol and nivalenol. World Mycotoxin J. 2010; 3(4):323-347. https://doi.org/10.3920/ WMJ2010.1247

12. Oshikata A, Takezawa, T. Development of an oxygenation culture method for activating the liver-specific functions of HepG2 cells utilizing a collagen vitrigel membrane chamber. Cytotechnology. 2015; 68(5):1801-1811. https://doi.org/10.1007/ s10616-015-9934-1

13. Pinton $P$, Oswald I. Effect of Deoxynivalenol and Other Type B Trichothecenes on the Intestine: A Review. Toxins. 2014; 6(5):1615-1643. https://www.ncbi.nlm.nih. gov/pmc/articles/PMC4052256/

14. Juan A, Berrada H, Font G, Ruiz M. Evaluation of acute toxicity and genotoxicity of DON, 3-ADON and 15-ADON in HepG2 cells. Toxicology Letters. 2017; 280S: S254-S266.
15. Kupcsik L. Estimation of Cell Number Based on Metabolic Activity: The MTT Reduction Assay. Mammalian Cell Viability. Methods Mol Biol. 2011; 740:13-19. https://doi. org/10.1007/978-1-61779-108-6 3

16. Jaimes $N$, Salmen $S$, Colmenares $M$, Burgos A, Tamayo L, Mendoza V, et al. Efecto citotóxico de los compuestos de inclusión de paladio (II) en la beta-ciclodextrina. Biomédica. 2016; 36(4):603-611. https:// doi.org/10.7705/biomedica.v36i4.2880

17. Dinu D, Bodea G, Ceapa C, Munteanu M, Roming $F$, Serban A, et al. Adapted response of the antioxidant defense system to oxidative stress induced by deoxynivalenol in Hek-293 cells. Toxicon. 2011; 57(78):1023-1032. https://doi.org/10.1016/j. toxicon.2011.04.006

18. Alassane I, Kolf M, Gauthier T, Abrami $R$, Abiola F, Oswald I. New insights into mycotoxin mixtures: the toxicity of low doses of Type $B$ trichothecenes on intestinal epithelial cells is synergistic. Toxicol Appl Pharmacol. 2013; 272(1):191-198. https:// doi.org/10.1016/j.taap.2013.05.023

19. Fernández C, Elmo L, Waldner T, Ruiz M. Cytotoxic effects induced by patulin, deoxynivalenol and toxin T2 individually and in combination in hepatic cells (HepG2). Food Chem Toxicol. 2018; 120:12-23. https://doi.org/10.1016/j.fct.2018.06.019

20. Lei Y, Guanghui Z, Xi W, Yingting W, Xialu $L$, Fangfang $Y$, et al. Cellular responses to T-2 toxin and/or deoxynivalenol that induce cartilage damage are not specific to chondrocytes. Sci Rep. 2017; 7(2231):1-14. https://www. nature.com/articles/s41598$\underline{017-02568-5}$

21. Mikami O, Yamaguchi $\mathrm{H}$, Murata $\mathrm{H}$, Nakajima Y, Miyazaki S. Induction of apoptotic lesions in liver and lymphoid tissues and modulation of cytokine mRNA expression by acute exposure to deoxynivalenol in piglets. J Vet Sci. 2010; 11(2):107-113. https://dx.doi. org/10.4142\%2Fjvs.2010.11.2.107

22. Ma Y, Zhang A, Shi Z, He C, Ding J, Wang $X$, et al. A mitochondria-mediated apoptotic pathway induced by deoxynivalenol in human colon cancer cells. Toxicol in Vitro. 2012; 26(3):414-420. https://doi.org/10.1016/j. tiv.2012.01.010 